\title{
Robust Optimization on the Fourth Party Logistics Network with Purchase - Return Reverse Logistics
}

\author{
Guo Haifeng \\ College of Automation and Electrical Engineering, Shenyang Ligong University,Shenyang, China \\ Email:ghf_1970@163.com
}

Keywords: fourth party logistics; reverse logistics; robust optimization; location-routing problem Abstract. One single-period many products 4PL network location model which synthetically taking the manufactory, centralized return centre, consumption area/initial return point, repair processing centre, distribution centre, and disposal centre into account,is constructed in this paper. The objective is to minimize the total cost. In the uncertain condition of recovery rate, sales and return rate, the model is contructed with the method of robust optimization. The result of the examples verified the robustness of the model.

\section{Introduction}

The concept of fourth party logistics(4PL) was proposed by Accenture Consulting in 1998. It is given in Strategic Supply Chain Alignment:'A 4PL is an integrator that assembles the resources,capabilities, and technology of its own organization and other organizations to design, build and run comprehensive supply chain solutions ${ }^{\text {[1] }}$.At present, the papers of 4PL path problems are based on multigraph to build models and focus on improving algorithm ${ }^{[2-6]}$. In this paper, the robust optimization is applied to 4PL.This paper will try to solve the problem is: to the minimum total cost as the goal, centralized return centre, repair processing centre and distribution centre in purchase-return the fourth party logistics network and then determine the optimal transport routes and numbers between the facilities, finally a numerical example is validated.

\section{PL network model with purchase-return reverse logistics}

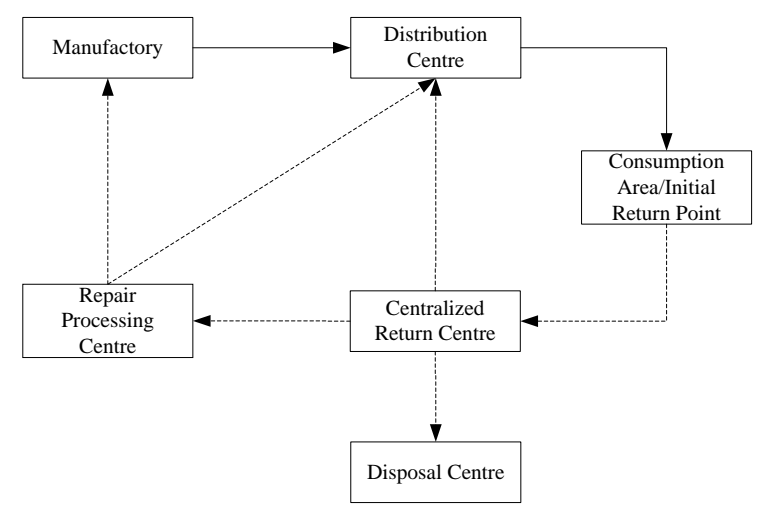

Fig. 1 Purchase-return 4PL network

Based on the references[],we construct a purchase-return 4PL network structure as shown in Fig. 1.It includes manufactory, centralized return centre, consumption area/initial return point, repair processing centre, distribution centre, and disposal center. All returned merchandise first ship from consuming area / initial return point to centralized return center, where going detection and classification: The damaged returns in the process of loading and unloading, transport and due to production and warranty period are shipped to repair processing centre, after dealing with necessary repair and restore its function to distribution center for sale again, while without restoring its function and damaged seriously goods shipped to manufactory for producing to restore its function again, then again shipped to distribution centers to wait for re-sale; due to reasons such as transportation, packaging, loading and unloading, goods which are sent by mistake are sent directly to distribution 
center for re-sale, at the same time to be replaced by the right goods, and re-transport to customers; due to reasons such as customers' personal preferences not defective "defects" are directly shipped to distribution centers, through simple operations such as cleaning ,maintenance and repackaging, continue to be saled in the market; while goods in centralized return centre which do not have function should be shipped to disposal center for landfill disposal. In order to facilitate analysis, we give the assumption and instructions are same as references[6,7] and appendix.

Then the model is as follows:

$$
\begin{aligned}
R_{s}= & \sum_{v=1}^{V} F_{v} \cdot y_{v} / T_{v}+\sum_{i=1}^{I} F_{i} \cdot y_{i} / T_{i}+\sum_{j=1}^{J} F_{j} \cdot y_{j} / T_{j}+\sum_{l=1}^{L} \sum_{i=1}^{I} \sum_{e=1}^{E} X_{s l i e} \cdot C_{l i e} \cdot d_{l i}+ \\
& \sum_{i=1}^{I} \sum_{j=1}^{J} \sum_{e=1}^{E} X_{s i j e} \cdot C_{i j e} \cdot d_{i j}+\sum_{i=1}^{I} \sum_{v=1}^{V} \sum_{e=1}^{E} X_{s i v e} \cdot C_{i v e} \cdot d_{i v}+\sum_{i=1}^{I} \sum_{k=1}^{K} \sum_{e=1}^{E} X_{s i k e} \cdot C_{i k e} \cdot d_{i k}+ \\
& \sum_{j=1}^{J} \sum_{v=1}^{V} \sum_{e=1}^{E} X_{s j v e} \cdot C_{j v e} \cdot d_{j v}+\sum_{j=1}^{J} \sum_{m=1}^{M} \sum_{e=1}^{E} X_{s j m e} \cdot C_{j m e} \cdot d_{j m}+\sum_{m=1}^{M} \sum_{v=1}^{V} \sum_{e=1}^{E} X_{s m v e} \cdot C_{m v e} \cdot d_{m v}+ \\
& \sum_{v=1}^{V} \sum_{l=1}^{L} \sum_{e=1}^{E} X_{s v l e} \cdot C_{v l e} \cdot d_{v l}+\sum_{v=1}^{V} \sum_{l=1}^{L} \sum_{e=1}^{E} X_{s v l e} \cdot C_{v e}+\sum_{l=1}^{L} \sum_{i=1}^{I} \sum_{e=1}^{E} X_{s l i e} \cdot C_{i e}+\sum_{i=1}^{I} \sum_{j=1}^{J} \sum_{e=1}^{E} X_{s i j e} \cdot C_{j e}+ \\
& \sum_{i=1}^{I} \sum_{k=1}^{K} \sum_{e=1}^{E} X_{s i k e} \cdot C_{k e}+\sum_{l=1}^{L} \sum_{i=1}^{I} \sum_{e=1}^{E} X_{s l i e} \cdot C_{l e}-\sum_{j=1}^{J} \sum_{m=1}^{M} \sum_{e=1}^{E} X_{s j m e} \cdot C S P_{m e}-\sum_{i=1}^{I} \sum_{j=1}^{J} \sum_{e=1}^{E} X_{s i j e} \cdot C S P_{j e}
\end{aligned}
$$

The objective function:

Constraints:

$$
\min \left\{\max _{s \in S}\left(R_{s}-O_{s}^{*}\right)\right\}
$$

$$
\begin{aligned}
& \sum_{i=1}^{I} X_{s l i e}=D_{l e} \cdot \alpha_{s e} \quad, \sum_{l=1}^{L} X_{\text {slie }}=\sum_{j=1}^{J} X_{s i j e}+\sum_{v=1}^{V} X_{s i v e}+\sum_{k=1}^{K} X_{s i k e}, \sum_{i=1}^{I} X_{s i j e}=\sum_{m=1}^{M} X_{s j m e}+\sum_{v=1}^{V} X_{s j v e}, \\
& \sum_{j=1}^{J} X_{\text {sjme }}=\sum_{v=1}^{V} X_{\text {smve }}, \sum_{l=1}^{L} X_{\text {svle }}=\sum_{m=1}^{M} X_{\text {smve }}+\sum_{j=1}^{J} X_{\text {sjve }}, \sum_{j=1}^{J} X_{\text {sije }}=\left(\beta_{e}+\gamma_{e}\right) \cdot \sum_{l=1}^{I} X_{\text {slie }} \text { ， } \\
& \sum_{v=1}^{V} X_{\text {sive }}=\left(\mu_{e}+\eta_{e}\right) \cdot \sum_{l=1}^{L} X_{s l i e}, \sum_{v=1}^{V} X_{s j v e}=\theta_{e} \cdot \sum_{i=1}^{I} X_{s i j e}, \forall s, i, j, v, l, e \\
& \sum_{l=1}^{L} X_{s l i e} \leq U_{i e} \cdot y_{i}, \sum_{i=1}^{I} X_{s i j e} \leq U_{j e} \cdot y_{j}, \sum_{l=1}^{L} X_{s v l e} \leq U_{v e} \cdot y_{v} \quad, \quad \forall s, i, j, v, e \\
& X_{\text {slie }} \leq w \cdot y_{i} \quad X_{\text {svle }} \leq w \cdot y_{v} \quad, X_{\text {sive }} \leq w \cdot y_{v}, X_{\text {sjve }} \leq w \cdot y_{v}, X_{\text {smve }} \leq w \cdot y_{v} \quad, X_{\text {sjve }} \leq w \cdot y_{j} \\
& X_{\text {sjme }} \leq w \cdot y_{j}, X_{\text {sije }} \leq w \cdot y_{j}, X_{\text {sike }} \leq w \cdot y_{i}, X_{\text {sive }} \leq w \cdot y_{i}, X_{\text {sije }} \leq w \cdot y_{i} \quad \forall s, i, j, k, l, m, v, e \\
& X_{\text {slie }}, X_{\text {sije }}, X_{\text {sive }}, X_{\text {sike }}, X_{\text {sjme }}, X_{\text {sjve }}, X_{\text {smve }}, X_{\text {svle }} \quad \forall s, l, i, j, k, m, v, e
\end{aligned}
$$

Where,constraints (1) show logistics conservation; constraints (2) say the maximum processing capacity of the facilities; constraints ( 3 ) say that only when the facilities are selected to have associated with traffic; $w$ represents infinity; constraints (4) limit the range of decision variables. 


\section{Numerical examples}

Table 1 The relevant data of consumption area/initial return point

\begin{tabular}{cccc}
\hline $\begin{array}{c}\text { Consumption } \\
\text { Area/Initial Return } \\
\text { Point } L_{l}\end{array}$ & $\begin{array}{c}\text { Position } \\
\text { Coordinates }\end{array}$ & $\begin{array}{c}\text { disposal cost per unit of } \\
\text { product } C_{l e}(\text { Yuan/Ton })\end{array}$ & Sales $D_{l e} \quad$ (Ton) \\
\hline$L_{1}$ & $(185,65)$ & $75 / 70$ & {$[800,1200] /[700,1250]$} \\
$L_{2}$ & $(125,80)$ & $60 / 55$ & {$[850,1200] /[900,1200]$} \\
$L_{3}$ & $(148,85)$ & $48 / 42$ & {$[700,900] /[800,900]$} \\
$L_{4}$ & $(110,70)$ & $80 / 76$ & {$[1100,1300] /[1200,1500]$} \\
\hline
\end{tabular}

To carry out effective sales - returns management, a large home appliance enterprise uses the above robust model, and build a high efficient and reasonable purchase - return fourth party logistics network. In this paper, the purchase - return situations of two kinds of products are analyzed. The location, disposal cost per unit of product and sales of consumption area/initial return point are shown in Table 1.

In order to facilitate analysis, other return behaviors are not considered (such as seasonal returns, cleaning inventory of retailers and product recall returns of manufacturers, etc.). According to the market situation, the return rates of major home appliances usually around $4.5 \%$, and the return rate of the components in the product life cycle may be much higher $(10 \%-25 \%)$. This numerical example assumes that return rate of product 1 is divided into 0.025 and 0.04 two kinds of cases, return rate of product 2 is divided into 0.03 and 0.04 two kinds of cases. These form 4 different scenarios. The specific combination of scenarios are shown in Table 2.

Table 2 combination of scenarios under uncertain

\begin{tabular}{ccc}
\hline scenario $S$ & return rate of product 1 & return rate of product 2 \\
\hline 1 & 0.025 & 0.03 \\
2 & 0.025 & 0.04 \\
3 & 0.04 & 0.03 \\
4 & 0.04 & 0.04 \\
\hline
\end{tabular}

The layout planning of centralized return centers, distribution centers and repair processing centers is the core content of the purchase - return fourth party logistics network.Determination of the alternative locations is not arbitrary, and we must follow the strategic principles such as adaptability, coordination, economy to choose appropriate addresses as candidate sites. By the comprehensive evaluation, $I_{1}, I_{2}, I_{3}$ and $I_{4}$ are selected as alternative locations of newly-bulit centralized return centers; $V_{1}, V_{2}$ and $V_{3}$ are selected as alternative locations of newly-bulit distribution centres; while considering the factors such as technology and resources, $J_{1}, J_{2}$ and $J_{3}$ are selected as alternative locations of newly-bulit repair disposal centres.All of these are shown in Table 3. 
Table 3 The relevant data of centralized return centre, repair processing centre and distribution centre

\begin{tabular}{ccccc}
\hline $\begin{array}{c}\text { alternative } \\
\text { facilities number } \\
I_{i} 、 V_{v} 、 J_{j}\end{array}$ & $\begin{array}{c}\text { alternative } \\
\text { facilities } \\
\text { position } \\
\text { coordinates }\end{array}$ & $\begin{array}{c}\text { fixed cost of new } \\
\text { facilities } F_{i} 、 F_{v} 、\end{array}$ & $\begin{array}{c}\text { disposal cost per unit of } \\
\text { product e } C_{i e} 、\end{array}$ & $\begin{array}{c}\text { the maximum } \\
\text { processing capacity }\end{array}$ \\
$F_{j}\left(10^{4 *}\right.$ yuan $)$ & $C_{v e} 、 C_{j e}$ (yuan/ton $)$ & $\begin{array}{c}U_{v e} 、 U_{j e} \\
\text { (ton) }\end{array}$ \\
\hline$I_{1}$ & $(100,40)$ & 25.5 & $100 / 110$ & $500 / 550$ \\
$I_{2}$ & $(120,70)$ & 30 & $120 / 110$ & $500 / 540$ \\
$I_{3}$ & $(165,75)$ & 25 & $150 / 135$ & $500 / 491$ \\
$I_{4}$ & $(125,90)$ & 20 & $125 / 110$ & $500 / 470$ \\
$V_{1}$ & $(125,115)$ & 15 & $80 / 75$ & $700 / 700$ \\
$V_{2}$ & $(145,130)$ & 15 & $75 / 70$ & $710 / 700$ \\
$V_{3}$ & $(160,100)$ & 18 & $82 / 80$ & $700 / 700$ \\
$J_{1}$ & $(120,100)$ & 30 & $150 / 145$ & $400 / 350$ \\
$J_{2}$ & $(150,105)$ & 38.8 & $140 / 142$ & $400 / 350$ \\
$J_{3}$ & $(140,120)$ & 25 & $150 / 140$ & $400 / 350$ \\
\hline
\end{tabular}

Table 4 The relevant data of known manufactory

\begin{tabular}{ccc}
\hline $\begin{array}{c}\text { manufactory } \\
\text { number } M_{m}\end{array}$ & $\begin{array}{c}\text { position } \\
\text { coordinates }\end{array}$ & $\begin{array}{c}\text { purchase costs per unit saved by manufactories for using recycled } \\
\text { product e per unit } C S P_{m e} \text { (yuan/ton) }\end{array}$ \\
\hline$M_{1}$ & $(125,130)$ & $100 / 150$ \\
$M_{2}$ & $(155,125)$ & $120 / 160$ \\
\hline
\end{tabular}

Purchase costs per unit saved by repair processing centres for using re-repaired product e per unit of alternative repair disposal centres are 110,120,130 and 145,150,160. Known position coordinates and purchase costs per unit saved by manufactories for using recycled product e per unit are shown in Table 4.Position coordinates and disposal cost per unit of product e of known disposal centers are shown in Table 5.

Table 5 The relevant data of known disposal centre

\begin{tabular}{ccc}
\hline number of disposal centers $K_{k}$ & $\begin{array}{c}\text { position } \\
\text { coordinates }\end{array}$ & $\begin{array}{c}\text { disposal cost per unit of product e } C_{k e} \\
\text { (yuan/ton) }\end{array}$ \\
\hline$K_{1}$ & $(130,75)$ & $80 / 90$ \\
$K_{2}$ & $(155,75)$ & $90 / 105$ \\
\hline
\end{tabular}

Due to different reasons of return, there is a certain difference about various probabilities of returns, and specific parameters are shown in table 6.

Table 6 various happening probabilities of returns

\begin{tabular}{cccccc}
\hline parameter & $\beta_{e}$ & $\gamma_{e}$ & $\mu_{e}$ & $\eta_{e}$ & $\theta_{e}$ \\
\hline & $0.15 / 0.1$ & $0.1 / 0.11$ & $0.05 / 0.04$ & $0.65 / 0.73$ & $0.5 / 0.45$ \\
\hline
\end{tabular}

In this numerical example, flexible and fast road return transportation is chosen,and calculation of shipping costs per unit of product e between facilities uses the relationship which unit transportation cost is proportional to the distance between facilities to measure. Unit transportation costs of two kinds of products are $C_{i j 1}=0.8 * d_{i j}$ and $C_{i j 2}=0.7 * d_{i j}$, and transportation distance is used Euclidean distance to express, that is $d_{i j}=\sqrt{\left(x_{j}-x_{i}\right)^{2}+\left(y_{j}-y_{i}\right)^{2}}$. The distances between facilities are shown in table 7. 
Table 7 distance between the facilities $(\mathrm{km})$

\begin{tabular}{cccccccccc}
\hline $\begin{array}{c}\text { facilities } \\
\text { number }\end{array}$ & $I_{1}$ & $I_{2}$ & $I_{3}$ & $I_{4}$ & $M_{1}$ & $M_{2}$ & $V_{1}$ & $V_{2}$ & $V_{3}$ \\
\hline$L_{1}$ & 88.60 & 65.19 & 22.36 & 65 & - & - & 78.10 & 76.32 & 43.01 \\
$L_{2}$ & 65 & 11.18 & 60.21 & 10 & - & - & 35 & 53.85 & 40.31 \\
$L_{3}$ & 65.80 & 31.76 & 19.72 & 23.54 & - & - & 37.80 & 45.10 & 19.21 \\
$L_{4}$ & 100 & 10 & 55.23 & 25 & - & - & 47.43 & 69.46 & 58.31 \\
$J_{1}$ & 20 & 30 & 51.48 & 15 & 30.41 & 43.01 & 15.81 & 39.05 & 40 \\
$J_{2}$ & 82.01 & 46.10 & 33.54 & 27.39 & 35.36 & 20.62 & 26.93 & 25.50 & 11.18 \\
$J_{3}$ & 89.44 & 53.85 & 51.48 & 32.02 & 18.03 & 15.81 & 15.81 & 11.18 & 28.28 \\
$K_{1}$ & 46.10 & 15 & 35 & 15.81 & - & - & - & - & - \\
$K_{2}$ & 65.19 & 35.36 & 10 & 33.54 & - & - & - & - & - \\
$V_{1}$ & 79.06 & 45.28 & 56.57 & 25 & 15 & 31.62 & - & - & - \\
$V_{2}$ & 100.62 & 65 & 58.52 & 44.72 & 20 & 11.18 & - & - & - \\
$V_{3}$ & 84.85 & 50 & 25.50 & 36.40 & 46.10 & 25.50 & - & - & - \\
\hline
\end{tabular}

Table 8 comparison of $\mathrm{O}^{*} \mathrm{~s}$ and Rs

\begin{tabular}{cccc}
\hline scenario $s$ & $R_{s}($ yuan $)$ & $O_{s}^{*}($ yuan $)$ & $\left(R_{s}-O_{s}^{*}\right) / O_{s}^{*}(\%)$ \\
\hline 1 & 350832.5 & 348729.5 & 5.99 \\
2 & 404453.5 & 402338.2 & 5.23 \\
3 & 439674.2 & 428046.2 & 5.54 \\
4 & 493295.2 & 490389.7 & 5.89 \\
\hline
\end{tabular}

To help the enterprise to find the optimal solution, we use lingo 10.0 software to solve the model in the paper. The optimal value of objective function is $z^{*}=11628$. Distribution centers are opened in 1,3 alternative location; centralized return centers are opened in 3,4 alternative location; repair processing centers are opened in 1 alternative location. In various scenarios, the amount of the optimal path and logistics data among the various facilities have nearly as many as a thousand, so this will not be listed. But, to illustrate robustness of constructed the fourth party logistics network model in sales and return rate in uncertainty, I have given the data of Table 8 . Table 8 shows comparison of Rs obtained with robust optimization in various scenarios and $\mathrm{O} * \mathrm{~s}$ under centain. We can see that the cost with robust optimization in various scenarios are generally higher than the cost in corresponding scenarios under certain, but deviation rate is within $6 \%$ in all scenarios. Because the fourth party logistics robust optimization model consider uncertainties in the worst case, it leads to the change of location and path of the fourth logistics netword. The results show that robust optimization method has been ideally control uncertainty of sales and return rate to make the purchase-return fourth party logistics network has better robustness.

\section{Conclusions}

As the e-commerce has become increasingly mature, returns management in the fourth party logistics has become an important problem to be solved in the consumer goods market, optimizing and designing purchase - return fourth party logistics network have very important economic significance. By building high-tech electronics purchase - return fourth party logistics location robust model, we use interval to describe uncertainty of sales and use unknown probability discrete scenarios to describe uncertainty of return rate, then we combine with a numerical example using the combination of interval analysis and scenario analysis method for the optimization of the model and obtain the optimum location strategy, path and logistics quantity. The numerical results demonstrate 
the robustness of the model and its solution, and have certain theoretical and practical value, and provide guidance for study of purchase - return fourth party logistics network optimization of high-tech electronic product.

\section{Acknowledgements}

This work is supported by National Natural Science Foundation (NNSF) of China under Grant(61402475).

\section{References}

[1] Foster T. 4PLs:the next generation for supply chain outsourcing?[J]. Logistics Management \& Distribution Report,1999,38(4):35.

[2] Gui Yunmiao,Gong Bengang,Cheng Youming. Robustness optimization and algorithm of supply chain network under uncertainty [J]. Statistics and Decision,2011,23(8): 172-174.

[3]Wang Baohua, He Shiwei. Robust Optimization Model and Algorithm for Logistics Center Locatio and Allocation under Uncertain Environment[J]. Journal of Transportation Systems Engineering and Information Technology, 2009, 9(2):69-74.

[4]Mir Saman Pishvaee,Masoud Rabbani,Seyed Ali Torabi.A robust optimization approach to closed-loop supply chain network design under uncertainty[J].Applied Mathematical Modelling, 2011,35(2):637-649.

[5]S.M.J. Mirzapour Al-e-hashem , H. Malekly , M. B. Aryanezhad. A Multi-objective Robust Optimization Model for Multi-product Multi-site Aggregate Production Planning In A Supply Chain Under Uncertainty[J]. Int. J. Production Economics, 2011, 34(1):28-42.

[6]Lin Qun, Guo Haifeng. Research on Recycling and Remanufacturing the Fourth Party Logistics Newwork Optimization[J].Journal of Shenyang Ligong University, 2012,31(3):1-6.

[7]Haifeng Guo, Qun Lin. Robust Optimization for the Location-routing of the 4PL Network Models with Reverse Logistics under the Uncertain Condition[C]. MIIT2014,10,7-8. 